\title{
Comportamiento de precios de las variedades de papa Parda Pastusa y Diacol Capiro en Colombia (1995-2011)
}

\section{Price performance of the potato varieties Parda Pastusa and Diacol Capiro in Colombia (1995-2011)}

\section{JUAN CARLOS BARRIENTOS 1,4}

CAMILO RONDÓN D. ${ }^{2}$

SANDRA E. MELO ${ }^{3}$

Cultivo de papa variedad Diacol

Capiro en la Sabana de Bogotá.

Foto: J.C. Barrientos

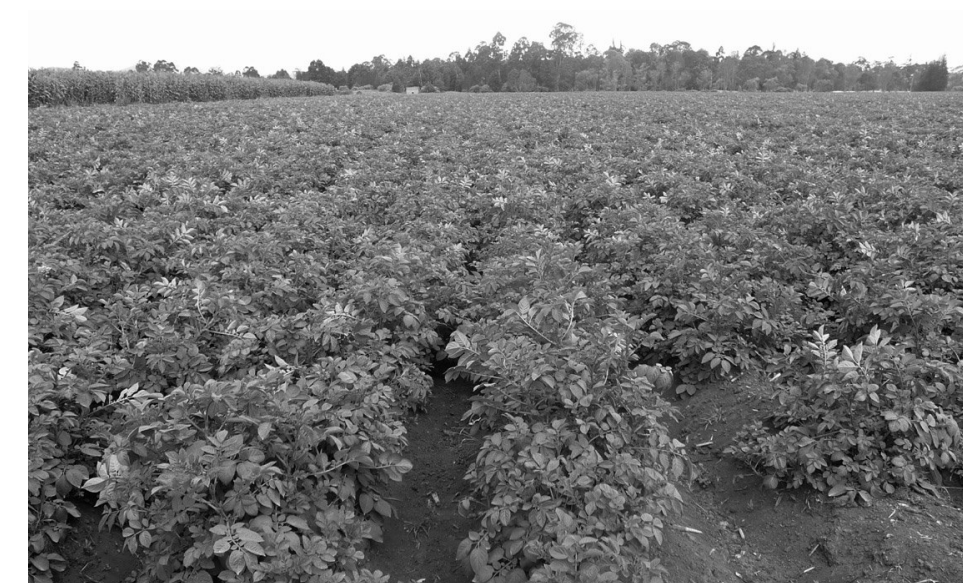

\section{RESUMEN}

El año 2013 los productores de papa de Colombia han protestado públicamente por la caída de los precios de este producto. Este fenómeno social no ha cambiado significativamente en los últimos años, pero cada año va agravándose afectando negativamente los ingresos de las familias productoras. Para hacer una aproximación explicativa se ha realizado una investigación documental sobre precios y un análisis estadístico de la serie de precios de las variedades Parda Pastusa y Diacol Capiro de los años 1995 a 2011. El análisis estadístico muestra resultados similares para ambas variedades. La tendencia de precios nominales es alcista y la de precios reales bajista. Las series presentan estacionalidad, siendo el primer semestre el de precios altos y el segundo el de precios medios y bajos. Este comportamiento estacional, sin embargo, tiene una ocurrencia de solo 65\% para el periodo de estudio. La variabilidad es alta a muy alta reflejando la amplitud de rango entre los precios máximos y mínimos. Esto representa un enorme riesgo para los productores, sobre todo para los pequeños que no tienen ningún seguro financiero para su producción. Las series de precios presentan ciclos irregulares; sin embargo, es posible desarrollar un modelo matemático de predicción de precios como el que se presenta aquí, un modelo Sarima desarrollado con el software estadístico R. Este permite hacer pronósticos para algunos meses, cuyos resultados ayudarían a planificar mejor las siembras. Junto a esta estrategia, también se puede recurrir a una mejor planificación del mercadeo y la innovación tecnológica, caminos que pueden reducir la variabilidad de precios ocasionada principalmente por el clima, plagas, enfermedades, y otros.

\footnotetext{
Facultad de Ciencias Agrarias, Departamento de Desarrollo Rural, Universidad Nacional de Colombia, Bogotá (Colombia). 2 Facultad de Ciencias Agrarias, Universidad Nacional de Colombia, Bogotá (Colombia).

3 Facultad de Ciencias Agrarias, Departamento de Agronomía, Universidad Nacional de Colombia, Bogotá (Colombia).

4 Autor para correspondencia. jcbarrientosf@unal.edu.co
} 
Palabras clave adicionales: tendencias, estacionalidad, variabilidad, ciclos, serie de tiempo.

\section{ABSTRACT}

During 2013, Colombian potato growers publicly protested the drop in potato prices. This price phenomenon has not significantly changed in recent years, but every year it worsens, adversely affecting the income of farming families. In order to provide an explanation, documentary research was conducted on the prices along with a statistical analysis of the price series of the potato varieties Parda Pastusa and Diacol Capiro from 1995 to 2011. The statistical analysis showed similar results for both varieties. The nominal price had an uptrend and the real prices had a downtrend. The series showed seasonality: the first semester had a tendency for high prices and the second one had middle to low prices. However, this seasonal performance only had an occurrence of $65 \%$ for the studied period. The price variability was high to very high, reflecting the breadth of the range between the maximum and minimum prices. This presents a huge risk for producers, especially for small farmers who have no financial insurance for their production. The price series had irregular cycles; however, it was possible to develop a mathematical model to predict the prices, a Sarima model, which was developed with the statistical software R. This model allows for price forecasting for some months. These results could help better plan seeding. Alongside this strategy, producers can use best marketing planning and technological innovation, methods that can reduce the price variability caused by weather, pests, and diseases, among others.

Additional key words: trends, seasonality, variability, cycles, time series.

Fecha de recepción: 20-05-2014

Aprobado para publicación: 10-11-2014
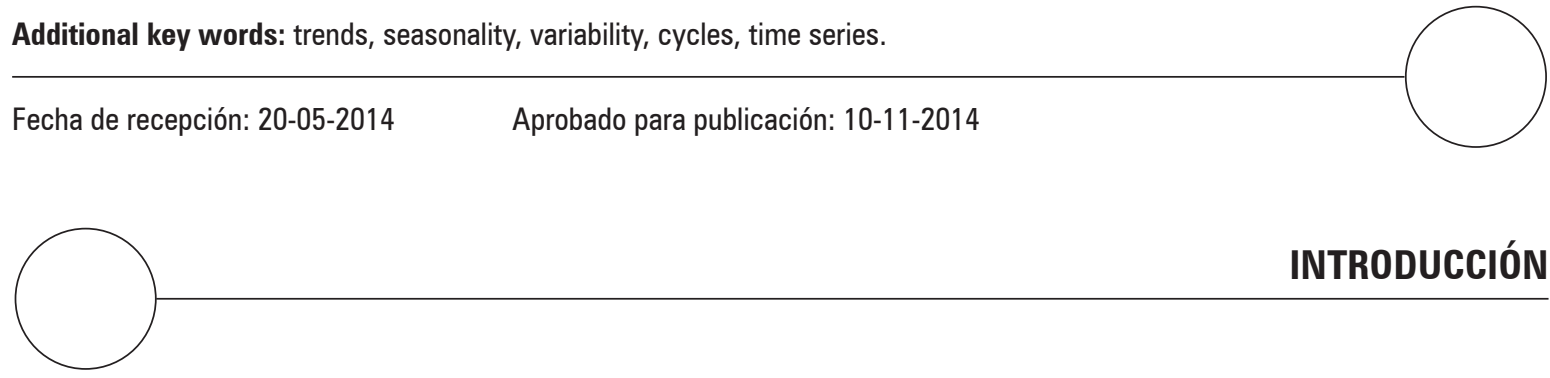

INTRODUCCIÓN

La papa es el cultivo más difundido y el producto más consumido a nivel mundial después del maíz, trigo y arroz. Se cultiva en 157 países. Según información relativamente actual, la producción mundial en 2010 alcanzó a algo más de 334 millones de toneladas (Faostat, 2013). Las regiones más productoras son Asia y Europa, y las de mayor consumo por persona son Europa y Norteamérica. En Latinoamérica la papa se cultiva en 25 países. En esta región el año 2007 Colombia ocupó el cuarto lugar en producción con 1.900.000 t después de Perú, Brasil y Argentina (FAO, 2009).

En Colombia, el cultivo de papa se encuentra entre los 10 más importantes del subsector de producción vegetal, y entre el primero y segun- do del grupo de los cultivos transitorios con 131.183 ha y 1.709.949 t de producción (Agronet, 2012; DANE 2011). Según H. Villarreal (comunicación personal, Consejo Nacional de la Papa, Bogotá, 2011) sobre generalidades del cultivo de papa en Colombia, para el año 2011 había aproximadamente 90.000 familias dedicadas al cultivo de la papa, generando además una gran demanda de semilla, fertilizantes, pesticidas y otros. En el territorio colombiano este cultivo se encuentra mayormente en la zona Andina, donde el clima es frío, las alturas mayores a $2.000 \mathrm{msnm}$, terrenos planos y en pendiente, y los suelos moderados a fértiles. Los departamentos con mayor producción en orden de importancia son: Cundinamarca, Boyacá, Nariño y Antioquia, de los cuales los dos pri- 
meros aportan cerca del 70\% de la producción nacional.

Colombia tiene 43 variedades de papa registradas, de las cuales 10 son comerciales; entre ellas se destacan Parda Pastusa y Diacol Capiro o R-12 negra. La primera se produce en toda la zona Andina de Colombia (2.900 a 3.400 $\mathrm{msnm}$ ) y se dirige principalmente para consumo en fresco; la segunda se produce en toda la zona papera (1.800 a $3.200 \mathrm{msnm})$, pero principalmente en Antioquia, y su destino es casi exclusivamente la agroindustria (Nústez, 2011). La variedad Parda Pastusa, sin embargo, está siendo reemplazada paulatinamente por la Pastusa Suprema, que tiene una presentación muy parecida, y es la que actualmente ocupa el primer lugar en producción y consumo a nivel nacional (Alba, 2012; Barrientos y Nústez, 2014).

Según Fedepapa (2004), hace una década la papa, como muchos otros alimentos, provenía principalmente de productores campesinos, pequeños productores con parcelas de menos de 3 ha, quienes constituían cerca del 90\% del total y contribuyeron con el $45 \%$ de la producción; los medianos (3-10 ha) hacían el 7\% de los productores y contribuían a la producción total con el 35\%; y los grandes (>10 ha) hacían solo el 3\%, pero aportaban con el $20 \%$ de la producción (MADR, 2005). Esa condición no ha cambiado significativamente en los últimos años. Por ejemplo, en Villapinzón, Cundinamarca, Sáenz et al. (2008) encontraron que la producción proveniente de parcelas menores a 3 ha cubrían el 48\% del total, parcelas entre 3 y 10 ha el $30 \%$ y parcelas mayores a 10 ha el 22\%; mostrando así una ligera tendencia a incrementar la proporción participativa de pequeños y grandes productores en la producción total.

La oferta de papa en Colombia depende casi en un $100 \%$ de la producción nacional, pues no hay importaciones significativas. Los factores determinantes de oferta son principalmente el clima, plagas y enfermedades, manejo del cultivo y planeación de siembras, precios de insumos y estructura de consumo. El clima está cambiando. Según Barrientos et al. (2009) el cambio climático en la zona papera y cebollera de Duitama, Boyacá, se ha estado manifestando, entre otros, con un incremento de la temperatura media y ampliación del rango de mínimos a máximos, así como con un incremento de la precipitación con reducción de frecuencia e incremento de la intensidad. Estas condiciones de cambio afectan la regularidad de la época de siembra así como propician un mayor ataque de enfermedades. Sin embargo, el manejo del cultivo no ha cambiado significativamente; el uso de semilla certificada todavía es reducido, la frecuencia de aplicaciones de pesticidas y el uso de fertilizantes aún no sigue un plan de eficiencia y eficacia (Alba, 2012; Barrientos y Nústez, 2011).

Por otra parte, la superficie de siembra de papa, que determina la cantidad de inversión, depende, entre otros, del último precio que recibió el productor; si fue bueno, siembra más, y si fue malo, siembra menos. Esto determina el volumen de oferta y el nivel de precios en el mercado (Corpoica, 2008). Según Franco (2005), el consumo de papa en Colombia es tradicional. El volumen de su compra depende de su calidad, la limpieza del lugar de venta y su precio. Sin embargo, los nuevos hábitos alimenticios y la variedad de sustitutos están propiciando una reducción en el consumo per cápita, que pasó de 76,4 kg por persona en 1995 a $58,0 \mathrm{~kg}$ por persona en 2008 (MADR 1995-2001, CNP 2002-2008; DANE en CNP, 2010). Según López (2013), más de 90\% de la producción se destina al consumo en fresco; pero Garay et al. (2010) sostienen que ya en 2008 el 12\% de la producción nacional se destinaba a la transformación, hojuelas y bastones, con tendencia a incrementarse.

De acuerdo con la teoría económica, los precios dependen de la interacción de oferta y deman- 
da. Según Confecampo (2008), el precio real recibido por los productores de papa en Colombia muestra una tendencia a la baja entre 1970 y 2005. Para esta institución, que se basó en la información de MADR (2005), el precio de la papa colombiana tiene alta volatilidad; los factores responsables para ella serían la estacionalidad de la producción, la alta perecebilidad del tubérculo, la inelasticidad del precio/demanda y la reducida capacidad de almacenamiento de la producción. Corpoica (2008) añadiría a ésta la oferta nacional, consecuencia de la superficie sembrada basada en el último precio recibido por el productor. Así mismo, se sostiene que el precio es estacional, donde sus máximos se ubican entre los meses de marzo y junio y los mínimos entre agosto y noviembre. En un trabajo realizado por el convenio SADE-Fedepapa (2009) sobre papa criolla, se corrobora la estacionalidad del precio, ubicándose los más altos en el primer semestre y los más bajos en el segundo; sin embargo, a pesar de que este comportamiento es similar en la mayoría de los años (1973-1993; 1994-2000 y 2008) y ciudades mencionadas (Bogotá, Cúcuta, Medellín y Tunja), no lo es siempre.

Por su parte, Bonilla et al. (2009) mencionan que los precios altos de papa Parda Pastusa entre 2004 y 2009 se han dado entre abril y junio/ julio y los bajos entre julio y enero. También llaman la atención sobre la gran variabilidad de los precios, factor que representa uno de los mayores riesgos para los productores, sobre todo para los pequeños. Por otro lado, Mosquera (2011) determinó mediante modelación que los precios de papa entre 2004 y 2009 de las variedades Pastusa Suprema, Diacol Capiro y Criollas en Pasto, Nariño, tienen un ciclo de 30 meses, es decir, al final de ese periodo la papa alcanza su precio más bajo o su precio más alto. Se puede decir de una manera general que el factor más influyente en los precios de la papa es la oferta, que a su vez depende de varias causas, que ya se han mencionado anteriormente (Rivas y Tapia, 2012).

\section{Planteamiento del problema}

En mayo de 2013, los productores de papa de Colombia, como poco antes habrían hecho ya los cafeteros, realizaron un paro acompañado de marchas y bloqueos de caminos. Su demanda al Gobierno tenía que ver con la caída del precio de la papa, que a muchos de ellos ya no les permitía sostener su trabajo e inversión. Si bien el gobierno otorgó un subsidio de 40.000 millones de pesos para paliar la crisis de este ramo productor en las siguientes dos cosechas, el problema aún no está solucionado, porque es estructural. Los precios del mercado, cuya oferta es preponderantemente nacional, seguirán siendo demasiado bajos para los productores (Portafolio, 2013; Sandoval, 2013). Este problema no es exclusivo de los colombianos, también lo sufren los productores de otros países como México, Argentina, Chile y Perú, entre otros.

Las causas son principalmente el incremento relativo estacional de la oferta debido a la concentración de la producción y venta, y la mejora de los rendimientos en los últimos años, así como la demanda inelástica y la disminución o muy bajo incremento de la demanda per cápita en fresco (Mosciaro, 2007; Proexpansión, 2011; Rivas y Tapia, 2012; Sabbagh et al, 2011). Las fuerzas del mercado colombiano de papa formadoras del precio han estado cambiando en detrimento de los productores, sobre todo de los pequeños.

Para hacer una aproximación explicativa a este fenómeno se tratará de responder a las siguientes preguntas: Cuál es la tendencia de los precios? Hay estacionalidad de precios? Se repite el comportamiento estacional todos los años? Es posible proyectar los precios? Qué perspectivas tiene el comportamiento de precios? En ese sentido el objetivo general de este trabajo es: realizar un análisis documental y estadístico del comportamiento de los precios de papa de las variedades Parda Pastusa y Diacol Capiro en Colombia entre los años 1995 y 2011. Los objetivos específicos 
son: determinar y analizar tendencias del precio; determinar y analizar rangos, estacionalidad, variabilidad y ciclicidad de los precios; ajustar un modelo que permita realizar predicciones de los precios; analizar los factores determinantes del comportamiento de precios.

\section{METODOLOGÍA}

Este trabajo se basa en una investigación documental y en un análisis estadístico. La investigación documental establece aportes explicativos de tipo inductivo, y gira alrededor del estudio "Análisis del comportamiento de precios de papa Parda Pastusa y Diacol Capiro en Colombia (1995-2011)" (Rondón, 2012), del cual se recogen los aspectos más sobresalientes. El análisis estadístico se basa en la serie de precios mensuales al productor $(\$ / \mathrm{kg})$ de 1995 a 2011 de las variedades Parda Pastusa (PP) y Diacol Capiro (DC) proporcionadas por el Consejo Nacional de la Papa. Se han escogido estas variedades por ser de las más demandadas a nivel nacional, la primera en fresco y la segunda para industria. La variedad Diacol Capiro presenta dos precios, uno como DC corriente, que es para consumo en fresco, y el otro como DC industrial, que es el destinado a transformación. Para el presente estudio se han tomado la serie de precios de DC industrial. Primero se realizó un análisis exploratorio y descriptivo de los datos, luego se buscó el modelo que mejor se ajustaba a los datos usando el criterio de información de Akaike (AIC). Los datos de precios, inicialmente nominales, se han convertido a precios reales descontando la inflación (IPC, año base 1998) de los años anteriores a 2011, el año base del presente estudio. Los resultados se presentan en gráficas y cuadros descriptivos. La interpretación y análisis se realiza fundamentado en una investigación documental del tema de comportamiento de precios agropecuarios. Posteriormente, a partir de la serie en estudio se ajustó un modelo Sarima (Brockwell y Davis, 1996; Peña, 2005) en el software estadístico R. Para ambas variedades, se utilizó el criterio de información Akaike (AIC) para el ajuste del modelo. Al realizar la prueba de hipótesis nula de una serie estacionaria contra la alternativa de la existencia de una raíz unitaria para las series diferenciadas se encuentra con un nivel de significancia $\alpha=0,05$ que no hay evidencia estadística para rechazar la hipótesis nula ya que el $P$-valor es de 0,1 ; satisfaciéndose así que las series estudiadas son estacionarias bajo el test de Kwiatkowski-PhillipsSchmidt-Shin. Los valores de ajuste fueron para 'Parda Pastusa' un AIC = 2.246 y para 'Diacol Capiro' AIC $=2.142$ con orden 2 en autorregresión y orden 2 en promedios móviles; se observó ausencia de tendencias en los residuos. Después, se realizó la validación del supuesto de normalidad bajo el test de Kolmogorov-Smirnov con un nivel de significancia de $\alpha=0,05$ y un valor de $P$ $=0,3391$ para 'Parda Pastusa' y $P=0.6121$ para 'Diacol Capiro'; y a un nivel de significancia de $\alpha=0,01$ bajo la prueba de Bartlett se obtuvo un valor $P=0,06305$ para 'Parda Pastusa' y $P=$ 0,14 para 'Diacol Capiro'. La prueba de homogeneidad de varianzas bajo el test de Levene arroja un valor $P=0,05011$ a un nivel de significancia de $\alpha=0,01$. El supuesto de independencia se puso a prueba bajo el test Box-Pierce con un resultado de valor $P=0,8322$ para 'Parda Pastusa' y $P=0,9036$ para 'Diacol Capiro'. Los resultados se presentan en ecuaciones y gráficas.

\section{RESULTADOS Y DISCUSIÓN}

\section{Las tendencias son alcistas en precios nominales y bajistas en precios reales}

A nivel general, la percepción que se tiene de los precios de alimentos, cuando estos son nominales, es que casi siempre que están subiendo año tras año. Esta percepción no es ajena al caso de la papa. Sin embargo, para poder determinar si la percepción es acertada o no, se elimina el factor de inflación y se obtienen los precios reales. 
Tendencia alcista cuando los precios son nominales

Los precios nominales, como ya se mencionó, muestran una tendencia alcista. Si bien los pre- cios de la variedad Diacol Capiro son algo menores al de Parda Pastusa, el comportamiento es similar, con oscilaciones que llegan hasta un rango de cerca de $1.200 \$ / \mathrm{kg}$ entre el mínimo y el máximo (figura 1). Se calcula de la tendencia,

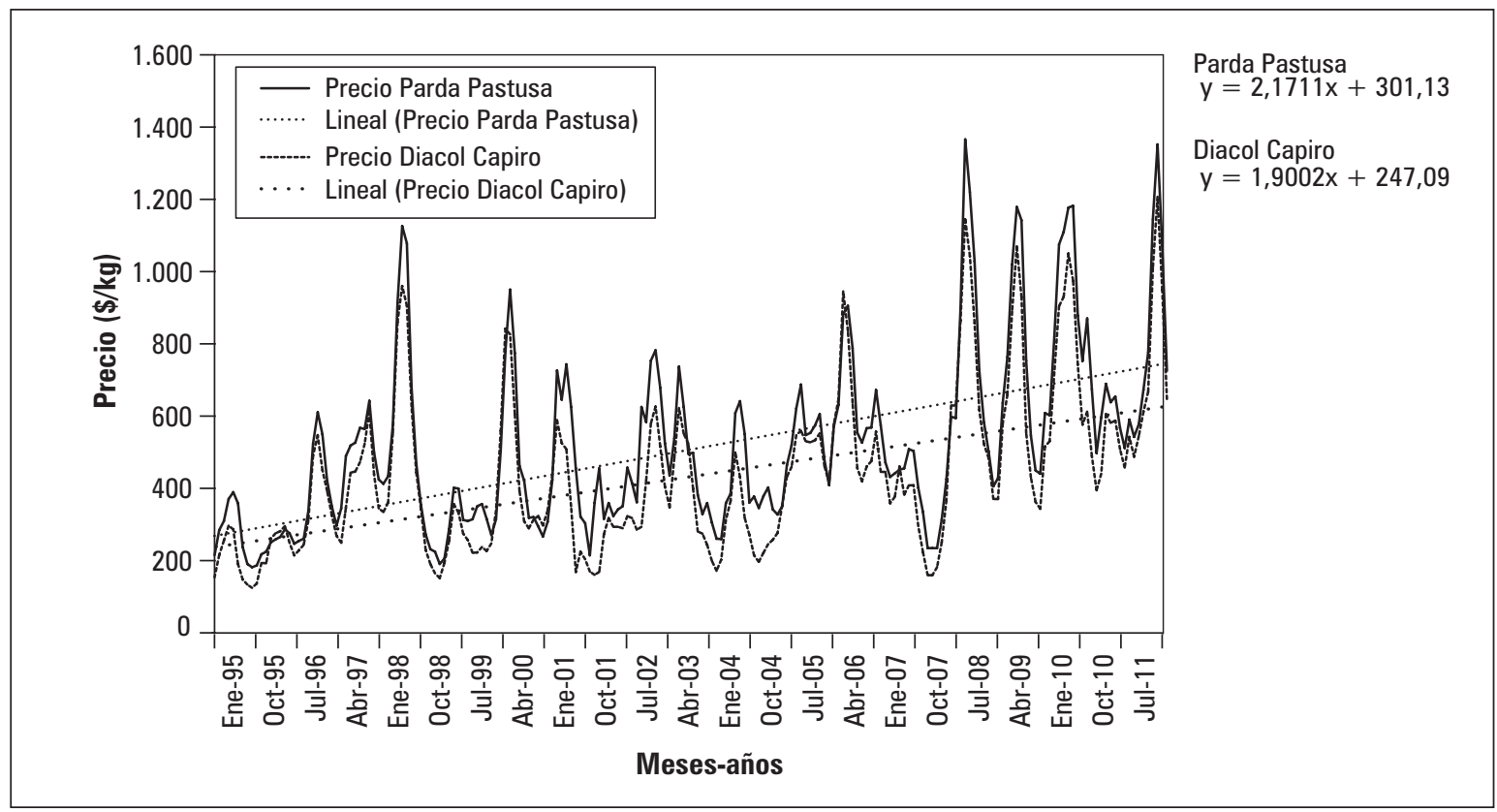

Figura 1. Tendencia de precios mensuales nominales $(\$ / \mathrm{kg})$ de papa variedades Parda Pastusa y Diacol Capiro para el periodo 1995-2011 en Colombia. Fuente: elaboración propia, a partir de datos de Consejo Nacional de la Papa, 2011.

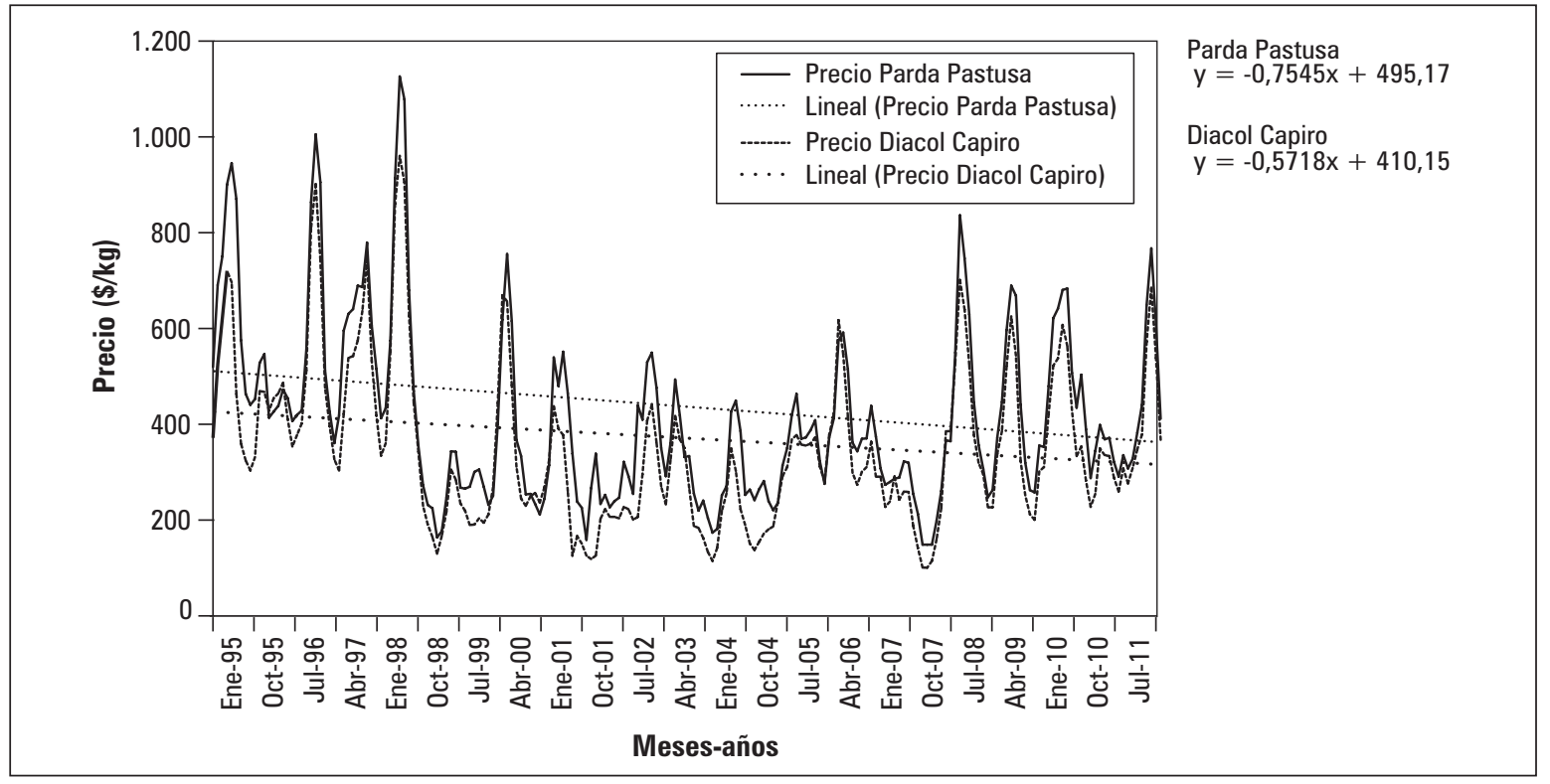

Figura 2. Tendencia de precios mensuales reales $(\$ / \mathrm{kg})$ de papa variedades Parda Pastusa y Diacol Capiro para el periodo 1995-2011 en Colombia. Fuente: elaboración propia, a partir de datos de Consejo Nacional de la Papa, 2011. 
que el incremento de precio para 'Parda Pastusa' es $0,72 \%$ mensual y $9,08 \%$ anual; mientras que para 'Diacol Capiro' es $0,76 \%$ mensual y $9,68 \%$ anual. Esa ligera diferencia puede deberse a un incremento de la demanda de la industria.

\section{Tendencia bajista cuando los precios son reales}

Contrario al comportamiento de los precios nominales, los precios reales tienden a bajar para ambas variedades (figura 2). La disminución de precio para Parda Pastusa es $0,15 \%$ mensual y $1,94 \%$ anual; mientras que para Diacol Capiro es $0,14 \%$ mensual y $1,77 \%$ anual. Si bien la disminución no parece grande, permite deducir que en promedio los precios se incrementan por debajo de la inflación. También en este caso se percibe que la variedad Diacol Capiro está en mejor condición que Parda Pastusa, porque la caída de sus precios es menor.

\section{Probabilidad de ocurrencia de tendencia anual "común"}

La tendencia "común" de los precios de papa en ambas variedades a lo largo del año es bajista; es decir, en el primer semestre se presentan los precios más altos y en el segundo los medios y bajos. La ocurrencia de esta tendencia anual es frecuente, pero no se da siempre. Se ha determi-

Tabla 1. Meses de precios altos y precios bajos, y tendencia anual de precios reales $(\$ / \mathrm{kg})$ de papa variedad Parda Pastusa de 1995 a 2011 en Colombia.

\begin{tabular}{|c|c|c|c|}
\hline Años & Meses de precios altos & Meses de precios bajos & Tendencia anual \\
\hline 1995 & Febrero - junio & Enero / julio - diciembre & Bajista \\
\hline 1996 & Octubre - diciembre & Enero - septiembre & Alcista \\
\hline 1997 & Mayo - noviembre & Enero - abril / diciembre & Alcista \\
\hline 1998 & Marzo - julio & Enero - febrero / agosto - diciembre & Bajista \\
\hline 1999 & Abril - noviembre & Enero - marzo / diciembre & Alcista \\
\hline 2000 & Febrero - mayo & Enero / junio - diciembre & Bajista \\
\hline 2001 & Febrero - mayo & Enero / junio - diciembre & Bajista \\
\hline 2002 & Agosto - diciembre & Enero - julio & Alcista \\
\hline 2003 & Enero / marzo - julio & Febrero/agosto - diciembre & Bajista \\
\hline 2004 & Abril - junio & Enero - marzo / julio - diciembre & Bajista \\
\hline 2005 & Abril - octubre & Enero - marzo / noviembre - diciembre & Alcista \\
\hline 2006 & Marzo - mayo / octubre & Enero - febrero / junio - septiembre / noviembre - diciembre & Bajista \\
\hline 2007 & Enero - julio & Agosto - diciembre & Bajista \\
\hline 2008 & Abril - julio & Enero - marzo / agosto - diciembre & Bajista \\
\hline 2009 & Febrero - junio / diciembre & Enero / julio - noviembre & Bajista \\
\hline 2010 & Enero - mayo / julio & Junio / agosto - diciembre & Bajista \\
\hline 2011 & Agosto - noviembre & Enero - julio / diciembre & Alcista \\
\hline
\end{tabular}

Fuente: elaboración propia, a partir de datos de Consejo Nacional de la Papa, 2011. 
Tabla 2. Meses de precios altos y precios bajos, y tendencia anual de precios reales $(\$ / \mathrm{kg})$ de papa variedad Diacol Capiro de 1995 a 2011 en Colombia.

\begin{tabular}{|c|c|c|c|}
\hline Años & Meses de precios altos & Meses de precios bajos & Tendencia anual \\
\hline 1995 & Febrero - mayo & Enero / junio - diciembre & Bajista \\
\hline 1996 & Octubre - diciembre & Enero - septiembre & Alcista \\
\hline 1997 & Junio - noviembre & Enero - mayo / diciembre & Alcista \\
\hline 1998 & Marzo - julio & Enero - febrero / agosto - diciembre & Bajista \\
\hline 1999 & Marzo - julio & Enero - febrero / agosto - noviembre & Alcista \\
\hline 2000 & Febrero - mayo & Enero / junio - diciembre & Bajista \\
\hline 2001 & Enero - mayo & Junio - diciembre & Bajista \\
\hline 2002 & Septiembre - diciembre & Enero - agosto & Alcista \\
\hline 2003 & Enero / marzo - julio & Agosto - diciembre & Bajista \\
\hline 2004 & Febrero - junio & Enero / julio - diciembre & Bajista \\
\hline 2005 & Mayo - octubre & Enero - abril / noviembre - diciembre & Alcista \\
\hline 2006 & Enero - mayo & Junio - diciembre & Bajista \\
\hline 2007 & Enero - junio & Julio - diciembre & Bajista \\
\hline 2008 & Abril - julio & Enero - marzo / agosto - diciembre & Bajista \\
\hline 2009 & Febrero - mayo / diciembre & Enero / junio - noviembre & Bajista \\
\hline 2010 & Enero - mayo & Junio - diciembre & Bajista \\
\hline 2011 & Septiembre - noviembre & Enero - agosto / diciembre & Alcista \\
\hline
\end{tabular}

Fuente: elaboración propia, a partir datos de Consejo Nacional de la Papa, 2011.

nado para ambas variedades una probabilidad de $65 \%$ de ocurrencia de la tendencia bajista y $35 \%$ alcista para el periodo de estudio (tablas 1 y 2).

Los precios son estacionales, de rangos amplios, alta variabilidad y ciclicidad irregular

Al realizar el análisis de la serie de precios de papa se pueden observar algunas estacionalidades. Con mayor frecuencia los precios de papa inician el año con valores bajos incrementándose hasta abril/mayo; esta sería una primera estacionalidad denominada de mayor incremento. Entre abril/mayo y agosto/septiembre hay una segunda estacionalidad denominada de mayor caída. Entre agosto/septiembre y octubre hay una tercera estacionalidad denominada de menor incremento. Finalmente, entre octubre y diciembre hay una cuarta estacionalidad denominada de menor caída (figuras 3 y 4). Este comportamiento estacional se debe al tiempo de las siembras que se realizan en la zona cundiboyancense, responsable de dos tercios de la producción nacional, donde se trata de aprovechar al máximo las lluvias para la formación y llenado de tubérculos. La primera gran siembra, cerca del $50 \%$ del total, se realiza a inicios de año, después de las probables heladas. La segunda siembra importante, cerca del $30 \%$, se reali- 


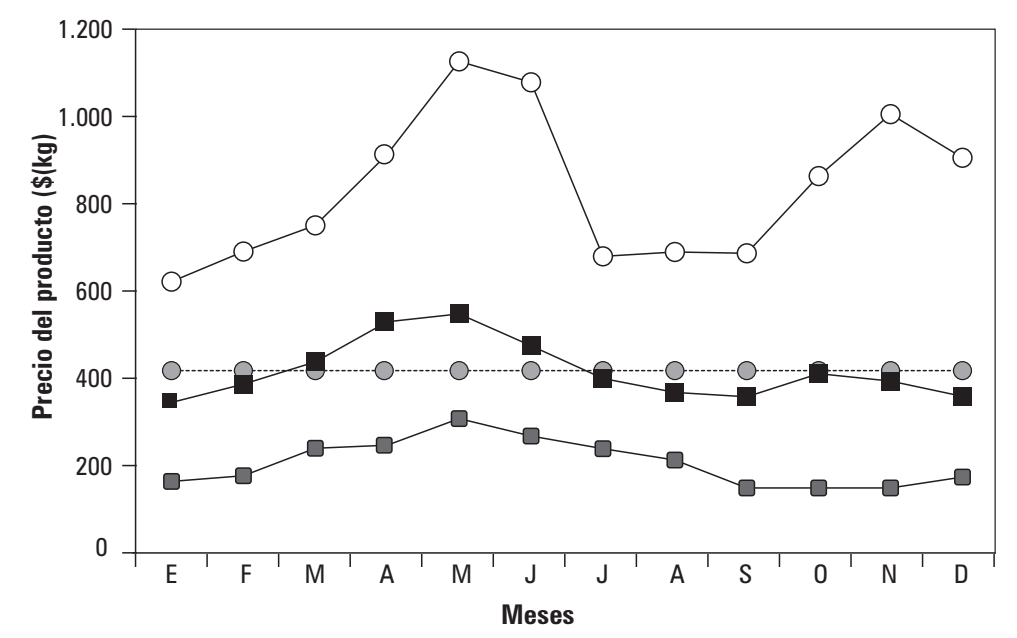

\begin{tabular}{|c|c|c|c|c|c|c|c|c|c|c|c|c|}
\hline - Pror & 345 & 387 & 438 & 530 & 548 & 475 & 400 & 368 & 358 & 411 & 394 & 359 \\
\hline D- Máximo mensual & 622 & 691 & 751 & 914 & 1127 & 1079 & 680 & 690 & 687 & 864 & 1006 & 906 \\
\hline ínir & 164 & 177 & 240 & 247 & 308 & 268 & 239 & 213 & 149 & 149 & 149 & 17 \\
\hline romedio & 418 & 418 & 418 & 418 & 418 & 418 & 418 & 418 & 418 & 418 & 418 & 41 \\
\hline
\end{tabular}

Figura 3. Comportamiento promedio de precios reales mensuales y anuales $(\$ / \mathbf{k g})$ de papa variedad Parda Pastusa para el periodo 1995-2011 en Colombia. Fuente: elaboración propia, a partir de datos de Consejo Nacional de la Papa, 2011.

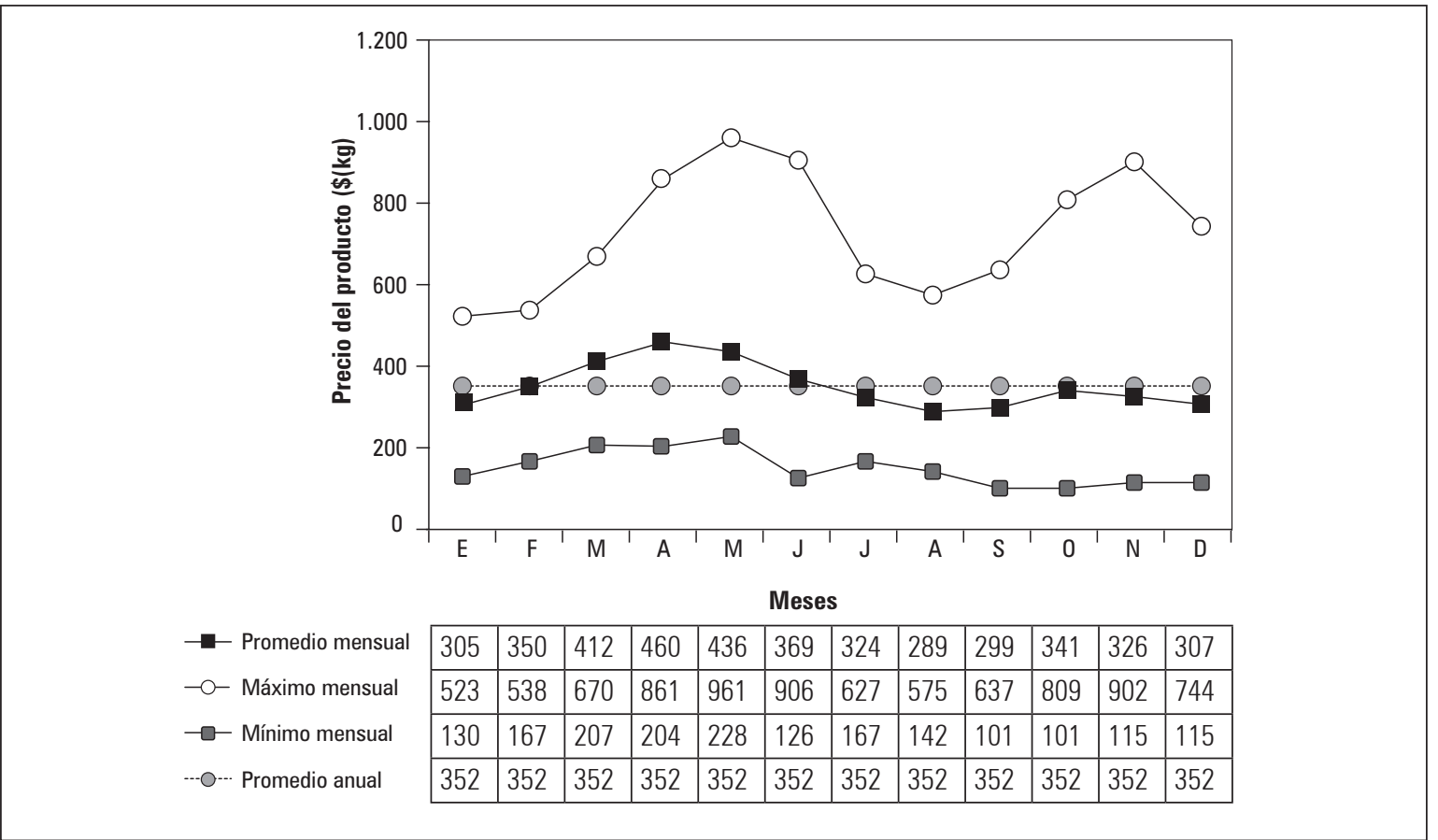

Figura 4. Comportamiento promedio de precios reales mensuales y anuales $(\$ / \mathrm{kg})$ de papa variedad Diacol Capiro para el periodo 1995-2011 en Colombia. Fuente: elaboración propia, a partir de datos de Consejo Nacional de la Papa, 2011. 
za a principios del segundo semestre. El restante $20 \%$ de la producción se siembra a lo largo del año (Corpoica, 2008; López, 2013).

Por otra parte, los precios máximos presentan una variación mayor que los precios mínimos respecto del precio promedio anual. Los rangos de precios (máximos-mínimos) más amplios se dan entre abril y junio y entre octubre y diciembre, siendo enero y julio los meses de menores rangos. El rango menor (441 $\$ / \mathrm{kg}$ ) se presenta en julio para 'Parda Pastusa' y en febrero (372 $\$ / \mathrm{kg}$ ) para 'Diacol Capiro'; el rango mayor se presenta en noviembre, 857 \$ kg para 'Parda Pastusa' y 787 \$/kg para 'Diacol Capiro'. La variación de precio puede ser en 'Parda Pastusa' de 1,1 a 2,1 veces mayor al precio promedio, mientras que en 'Diacol Capiro' puede ir de 1,1 a 2,2 veces. La variabilidad de precios es grande, de 100 a 200\% durante el mismo mes, es decir, una variabilidad alta a muy alta. Adicionalmente, se presentan ciclos de precios de forma irregular; según López (2013), de dos, tres y cinco años como indican los resultados de un estudio del periodo 1999 a 2012.

\section{Es posible predecir precios mediante un mo- delo matemático}

Con los precios reales de las dos variedades de papa bajo estudio, deflactados mensualmente, se han desarrollado modelos Sarima (figuras 5 y $6)$, que permiten hacer predicciones de 4 a 5 me- ses, es decir, antes de comenzar un nuevo ciclo de producción. Esto puede permitir a productores y otros agentes de la cadena de papa aproximarse a posibles precios y estados de mercado antes de decidir sobre la próxima siembra. Los modelos presentan periodicidades de 6 y 12 meses, siendo esta última la que corrobora la estacionalidad promedio vista anteriormente.

El modelo SARIMA $(2,1,2)(2,1,2)_{12}$ ajustado para la variedad Parda Pastusa es:

$$
\begin{aligned}
& \mathrm{Y}_{t}=1.3644 Y_{t-1}-0.643 Y_{t-2}-0.4287 Y_{t-12}- \\
& 0.3083 Y_{t-24}+1.0706 \mathrm{e}_{t-1}-0.1362 \mathrm{e}_{t-2}+0.4055 \mathrm{e}_{t-} \\
& { }_{12}+0.2566 \mathrm{e}_{t-24}+\mathrm{e}_{t}
\end{aligned}
$$

Donde $\mathrm{e}_{t} \sim N(0,6250)$.

A continuación se presentan cuatro predicciones de precio de la variedad Parda Pastusa para los meses siguientes (enero-abril) bajo el modelo ajustado usando intervalos de confianza del $95 \%$.

El modelo escogido es bueno en su ajuste. Al usar el criterio de Akaike, presentó el menor valor comparando con otros modelos probados. $\mathrm{Su}$ valor fue de un $\mathrm{AIC}=2246$ y $\mathrm{AIC}=2142$ respectivamente. Se consideró un orden de 2 en la parte autorregresiva y también de orden 2 en los promedios móviles; además, se diferenció una vez la serie para lograr estacionariedad. Con el

Tabla 3. Predicciones de precios para variedad Parda Pastusa usando modelo Sarima $(2,1,2)(2,1,2)_{12}$

\begin{tabular}{|c|c|c|}
\hline $\begin{array}{c}\text { Intervalos de confianza al 95\% para } \\
\text { las predicciones }\end{array}$ & $\begin{array}{c}\text { Estimación puntual precio 'Parda } \\
\text { Pastusa' }(\$ / \mathrm{kg})\end{array}$ & $\begin{array}{c}\text { Error estándar } \\
232.0520 ; 390.1750\end{array}$ \\
\hline $150.9704 ; 409.5327$ & 311.1135 & 129.2811 \\
\hline $174.6259 ; 494.2008$ & 280.2526 & 159.7875 \\
\hline $272.7120 ; 619.6580$ & 334.4134 & 173.4730 \\
\hline
\end{tabular}


Tabla 4. Predicciones de precios para variedad Diacol Capiro usando modelo Sarima $(2,1,2)(2,1,2)_{12}$

\begin{tabular}{|c|c|c|}
\hline $\begin{array}{c}\text { Intervalos de confianza al 95\% para } \\
\text { las predicciones }\end{array}$ & $\begin{array}{c}\text { Estimación puntual precio 'Diacol } \\
\text { Capiro' }(\$ / \mathrm{kg})\end{array}$ & \begin{tabular}{c} 
Error estándar \\
\hline $206.6019 ; 325.1383$
\end{tabular} \\
\hline 127,$6361 ; 331,6025$ & 265.8701 & 101.98322 \\
\hline $164.4776 ; 423.2587$ & 229.6193 & 129.39054 \\
\hline $220.6152 ; 504.6457$ & 293.8682 & 142.01524 \\
\hline
\end{tabular}

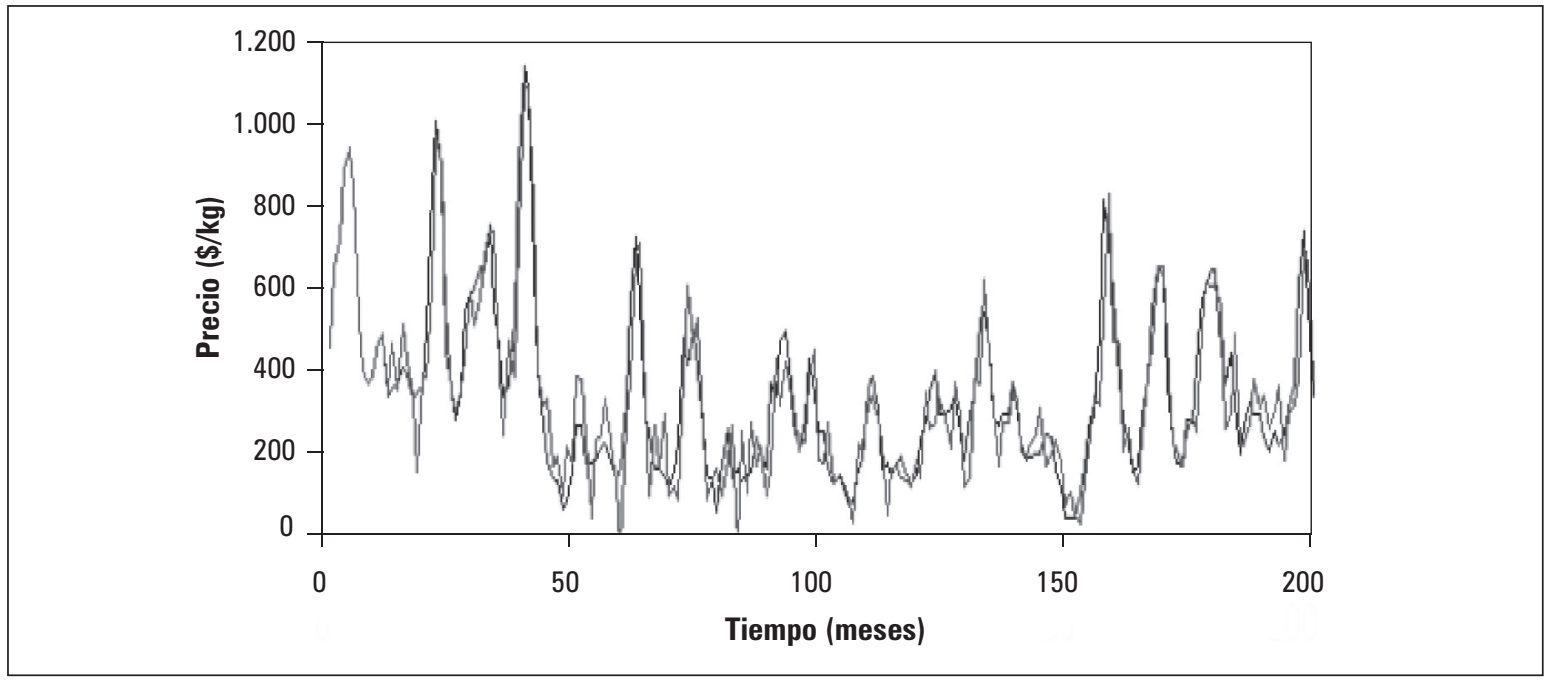

Figura 5. Serie original deflactada y modelo Sarima ajustado de precios para la variedad de papa Parda Pastusa (1995-2011).

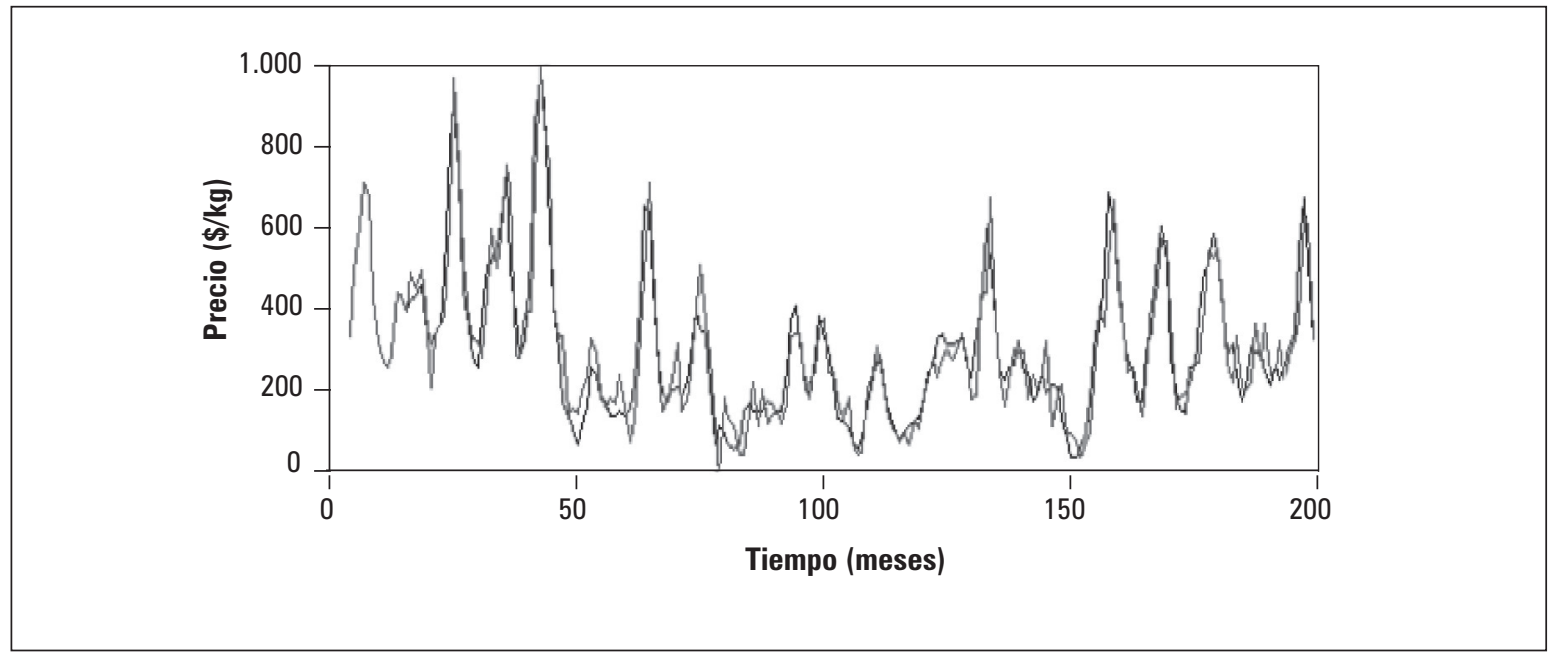

Figura 6. Serie original deflactada y modelo Sarima ajustado de precios para la variedad de papa Diacol Capiro (1995-2011). 
periodograma se encontró un estacionalidad de periodicidad cada 12 meses.

Modelo SARIMA $(2,1,2)(2,1,2)_{12}$ ajustado para la variedad Diacol Capiro es:

$$
\begin{aligned}
& \mathrm{Y}_{t}=1.3765 \mathrm{Y}_{t-1}-0.6939 Y_{t-2}-0.7414 Y_{t-12}- \\
& 0.3510 Y_{t-24}+0.976 \mathrm{e}_{t-1}-0.0859 \mathrm{e}_{t-2}+0.2488 \mathrm{e}_{t-} \\
& { }_{12}+0.5093 \mathrm{e}_{t-24}+\mathrm{e}_{t}
\end{aligned}
$$

Donde $\mathrm{e}_{t} \sim N(0,3507)$.

A continuación se presentan cuatro predicciones de precio de papa 'Diacol Capiro' para los meses siguientes (enero-abril) bajo el modelo ajustado usando intervalos de confianza del $95 \%$.

La serie ajustada, que se muestra de color azul en las figuras 5 y 6 presenta valores muy cercanos a los reales (color negro) debido a que el ajuste del modelo es bueno, lo cual lo hace apropiado para realizar predicciones.

\section{Los factores más determinantes del comportamiento de precios de la papa}

\begin{tabular}{|c|c|c|c|}
\hline Oferta & \multicolumn{2}{|c|}{ Precios } & Demanda \\
\hline $\begin{array}{l}\text { Precio del periodo anterior } \\
\text { Disponibilidad de semilla } \\
\text { Disponibilidad de tierra } \\
\text { Disponibilidad de capital financiero } \\
\text { Costos de producción }\end{array}$ & $\begin{array}{l}\text { Superficie de } \\
\text { cultivo }\end{array}$ & $\begin{array}{l}\text { Consumo de papa } \\
\text { fresca }\end{array}$ & $\begin{array}{l}\text { Gustos y preferencias de } \\
\text { consumidor } \\
\text { Consumo de productos } \\
\text { substitutos } \\
\text { Ingresos del consumidor }\end{array}$ \\
\hline $\begin{array}{l}\text { Clima: precipitación, temperatura, etc. } \\
\text { Suelo } \\
\text { Tecnología: variedades, sistemas de } \\
\text { producción, planificación, manejo, etc. } \\
\text { Ataque de enfermedades y plagas }\end{array}$ & Rendimiento & \multirow[t]{2}{*}{$\begin{array}{l}\text { Consumo de materia } \\
\text { prima para industria }\end{array}$} & \multirow{2}{*}{$\begin{array}{l}\text { Consumo de alimentos } \\
\text { procesados } \\
\text { Consumo de productos } \\
\text { procesados no } \\
\text { alimenticios } \\
\text { Innovaciones industriales }\end{array}$} \\
\hline $\begin{array}{l}\text { Capacidad de almacenamiento } \\
\text { Poder de mercado }\end{array}$ & \multirow[t]{2}{*}{ Intermediación } & & \\
\hline Control & & \multirow[b]{2}{*}{ Intermediación } & \multirow{2}{*}{$\begin{array}{l}\text { Capacidad de } \\
\text { almacenamiento } \\
\text { Poder de mercado } \\
\text { Control }\end{array}$} \\
\hline $\begin{array}{l}\text { Estrategias } \\
\text { Instrumentos: producto, precio, } \\
\text { comunicación, distribución, etc. }\end{array}$ & Mercadeo & & \\
\hline $\begin{array}{l}\text { Producto fresco } \\
\text { Producto transformado }\end{array}$ & Importaciones & Exportaciones & $\begin{array}{l}\text { Producto fresco } \\
\text { Producto transformado }\end{array}$ \\
\hline
\end{tabular}

De las fuerzas que establecen los precios de papa, que son oferta y demanda, la mayoría

Figura 7. Factores de oferta y demanda que determinan los precios de papa. 
de los autores le atribuyen mayor influencia a la oferta, debido a que sus factores determinantes cambian con mayor frecuencia y tienen efectos notables a corto plazo. Aunque no se sabe con certeza el grado de influencia que cada uno ejerce, los factores más relevantes de la oferta son el clima (fenómenos Niño y Niña, cambio climático), los precios del periodo anterior, tecnología de producción, los costos de producción y el ataque de enfermedades y plagas (figura 7).

Adicionalmente, la disponibilidad de agua (de lluvia) y la ocurrencia de heladas (temperaturas bajas) determinan la estacionalidad de precios y el rendimiento. Los costos de producción y los precios buenos o malos del periodo anterior (información desactualizada y no proyectada) determinan las superficies de siembra; y el clima, las plagas y enfermedades el rendimiento.

El papel de la intermediación es regular volúmenes de venta, pero también puede especular con los precios. La importación y la exportación aún no son relevantes, y en consecuencia su influencia en el precio nacional tampoco. La demanda aparentemente se ajusta a la oferta. A mediano y largo plazo la tendencia del consumo en fresco es a la baja y la de la industria al alza. A pesar de todo esto, hay factores que pueden ser manejados a favor de precios más estables. Estos serían: el mercadeo, la generación y difusión de información de mercado, la planificación de la producción, la innovación tecnológica a nivel de finca, industria y consumo. Para su implementación, sin embargo, se requerirían importantes recursos financieros (Burgos, 2009; Casparri, 2012; CEPAL/FAO/ IICA, 2011; FAO, 2010; García, 2007; Infoagro, 2013; López, 2013; Mosciaro, 2007; Pérez, 1985; Rivas y Tapia, 2012; Rojas, 2011). Según Villarreal (2012), el Acuerdo de Competitividad de la cadena de papa apunta en este sentido.

\section{CONCLUSIONES Y RECOMENDACIONES}

La serie analizada presenta una tendencia a la baja en precios reales, estacionalidad, variabilidad alta y ciclicidad irregular; también permite desarrollar un modelo de pronósticos. Ambas variedades presentan características similares. La tendencia en precios nominales es alcista, aproximadamente $10 \%$ anual; y bajistas en precios reales, aproximadamente $2 \%$ anual.

Los precios más altos se presentan en el primer semestre y los medios y bajos en el segundo semestre. Sin embargo, este comportamiento tiene una probabilidad de ocurrencia de solo $65 \%$. Los precios presentan estacionalidad con cuatro estaciones claramente marcadas. Los rangos, diferencia entre precio máximo y mínimo, son amplios y expresan un alta variabilidad, que representa un alto riesgo para los productores, sobre todo para los pequeños. La serie presenta ciclos de dos, tres y cinco años, que denotan irregularidad. Sin embargo, la serie se ajustó a un modelo Sarima, que permite hacer predicciones de máximo 5 meses bajo un 95\% de confianza. Los factores determinantes del comportamiento de precios de la papa son aquellos relacionados principalmente con la oferta como son el clima, precios del periodo anterior, tecnología de producción, costos de producción, intermediación, y el ataque de enfermedades y plagas. La planificación de la producción y el mercadeo, así como la innovación tecnológica a nivel de finca, industria y consumo pueden disminuir la volatilidad de precios. Estas últimas son estrategias que pueden ser implementadas poco a poco; aunque requieren recursos financieros.

Para posteriores investigaciones se recomienda profundizar en temas relacionados con el análisis de precios y utilidades del productor papero, gestión de la información de precios, planificación de la producción, entre otros. 


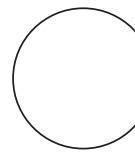

\section{REFERENCIAS BIBLIOGRÁFICAS}

Agronet. 2012. Estadísticas de producción nacional de papa. En: http://www.agronet.gov.co; consulta: noviembre de 2012 .

Alba, A.F. 2012. Análisis de la unidad productiva y del manejo de insumos en el cultivo de papa (Solanum tuberosum L.) en el departamento de Boyacá. Trabajo de grado. Facultad de Agronomía, Universidad Nacional de Colombia, Bogotá.

Barrientos, J.C. y C.E. Nustez. 2014. Difusión de seis nuevas variedades de papa en Boyacá y Cundinamarca (Colombia) entre 2003 y 2010. Rev. Colomb. Cienc. Hortic. 8(1), 126-141.

Barrientos, J.C. y C.E. Nústez. 2011. Evaluación del impacto socioeconómico de nuevas variedades de papa en Boyaca y Cundinamarca, Colombia. Informe. Proyecto Red de innovación de investigación y desarrollo: hacia la diseminación eficiente y mecanismos de impacto pro-pobre con nuevas variedades de papa en la zona andina. Universidad Nacional de Colombia - Fontagro, Bogotá.

Barrientos, J.C., G.A. Leyva y J.C. Morales. 2009. Efectos del cambio climático sobre la oferta y precios de cebolla de bulbo. Caso del municipio Duitama, Boyacá (Colombia). Rev. Colomb. Cienc. Hortic. 3(1), 69-80.

Bonilla, M.H., F. Cardozo y A. Morales. 2009. Agenda prospectiva de investigación y desarrollo tecnológico para la cadena productiva de la papa en Colombia con énfasis en papa criolla. Ministerio de Agricultura y Desarrollo Rural (Proyecto Transición de la Agricultura); Universidad Nacional de Colombia; Corpoica, Bogotá.

Burgos, E. 2009. Análisis de la volatilidad de los precios agrarios de productos perecederos: aplicación a los sectores del fresón y de los cítricos. Trabajo final. Escuela Técnica Superior de Ingenieros Agrónomos, Universidad Politécnica de Madrid, Madrid.

Casparri, M.T., A. Bernardello, J. García y A.S. Vilker. 2012. Impacto económico-financiero y actuarial del riesgo climático en Argentina. Facultad de Ciencias Económicas, Universidad de Buenos Aires, Buenos Aires.

CEPAL/FAO/IICA. 2011. Volatilidad de precios en los mercados agrícolas (2000-2010): implicaciones para América Latina y opciones de políticas. Boletín 1/2011. Santiago.
CNP. 2010. Tabla 41. Evolución del consumo aparente y per cápita de papa en Colombia, 1995-2008. Consejo Nacional de la Papa, Bogotá.

Confecampo. 2008. Análisis del mercado de la papa en Colombia. Departamento Técnico, Confederación Empresarial del Campo de Colombia, Bogotá.

Corpoica. 2008. Factores que determinan el incremento en el precio de la papa. 4p. En: http://corpomail.corpoica.org.co/BACFILES/BACDIGITAL/55508/55508. pdf; consulta: 10 de octubre de 2013.

DANE. 2011. Resultados Encuesta Nacional Agropecuaria (ENA). Departamento Administrativo Nacional de Estadística, Bogotá.

Brockwell, J.P. y R.A. Davis. 1996. Introduction to time series and forecasting. Springer, New York, NY.

FAO. 2010. La volatilidad de precios en los mercados agrícolas. Perspectivas económicas y sociales. Informes de Política 12. FAO, Roma.

FAO. 2009. Año internacional de la papa 2008: Nueva luz sobre un tesoro enterrado. FAO, Roma.

Faostat. 2013. Dirección de estadística. En: http://faostat.fao.org/DesktopDefault. aspx?PageID=567\&lang=es\#ancor; consulta: 22 de julio de 2013.

Fedepapa. 2004. Guía ambiental para el cultivo de la papa. Boletín diagráfica para el Ministerio de Ambiente, Vivienda y Desarrollo Territorial, Bogotá.

Franco, N. 2005. Estudio básico sobre consumo de papa en hogares de Colombia. Ipsos. Documento preparado para Cevipapa. Bogotá.

Garay, L.J., F. Barberi e I. Cardona L. 2010. Impactos del TLC con Estados Unidos sobre la economía campesina en Colombia. Instituto Latinoamericano para una Sociedad y un Derecho Alternativos (ILSA); Oxfam, Bogotá.

García, M. 2007. Análisis del comportamiento de precios de cinco productos hortícolas en Costa Rica de 1999 al 2003. Tecnología en Marcha 21(2), 30-44.

Infoagro. 2013. Informe sobre el comportamiento de precios. Ministerio de Agricultura de Colombia 29/11/2010. En: http://www.infoagro.com/noticias/2010/11/17133 informe comportamiento precios.asp; consulta: 10 de noviembre de 2013 
López, E. 2013. El mercadeo y los precios de la papa. Rev. Papa 29, 36-39.

MADR. 2005. La cadena de la papa en Colombia: una mirada global de su estructura y dinámica 19912005. Ministerio de Agricultura y Desarrollo Rural; Observatorio Agrocadenas Colombia. Documento de trabajo No. 54. Bogotá.

Mosciaro, M. 2007. Comportamiento del precio de la papa en el mercado mayorista argentino. Argenpapa, Argentina, 18-09-2007. En: http://www.argenpapa.com.ar/?id=1358; consulta: 10 de noviembre de 2013.

Mosquera, F.A. 2011. Diseño de un modelo de planificación de cultivos para mejorar el desempeño de pequeños y medianos productores de papa en el municipio de Pasto - Departamento de Nariño. Tesis de maestría. Facultad de Ingeniería, Universidad de la Sabana, Chía, Colombia.

Nústez, C.E. 2011. Variedades colombianas de papa. Facultad de Agronomía, Universidad Nacional de Colombia, Bogotá.

Peña, D. 2005. Análisis de series temporales. Alianza Editorial, Madrid.

Pérez, F. 1985. Estabilización de precios agrícolas: un enfoque financiero. Universidad Católica de Chile, Santiago.

Portafolio. 2013. Paperos recogen la estrategia de los cafeteros con paro. Portafolio, negocios, Mayo 8 de 2013 - 10:27 pm. En: http://www.portafolio. $\mathrm{co} /$ negocios/paro-los-paperos-colombia; consulta: 10 de diciembre de 2013.

Proexpansión. 2011. Cambios del sector papa en el Perú en la última década: Los aportes del proyecto Innovación y Competitividad de la Papa (Incopa). Centro Internacional de la Papa (CIP), Lima.
Rivas, T. y B. Tapia. 2012. Estructura de mercado y determinantes del precio de la papa para consumo en fresco. Oficina de Estudios y Políticas Agrarias (ODEPA); Ministerio de Agricultura, Santiago.

Rojas, E.O. 2011. Evaluación del desarrollo del cultivo de papa bajo escenarios de variabilidad climática interanual y cambio climático, en el sur oeste de la Sabana de Bogotá. Tesis de maestría. Facultad de Ciencias, Universidad Nacional de Colombia, Bogotá.

Rondón, C. 2012. Análisis del comportamiento de precios de papa Parda Pastusa y Diacol Capiro en Colombia (1995-2011). Trabajo de grado. Facultad de Agronomía, Universidad Nacional de Colombia, Bogotá.

Sabbagh, A., J.A. García, J.A. Matus, L. Jiménez y M. Hernández. 2011. Comportamiento del consumo de papa (Solanum tuberosum L.) fresca en México. Rev. Mex. Cienc. Agríc. 2(4), 559-572.

Sáenz H., M.R. Piña y S. Roa. 2008. Diseño y puesta en marcha de negocios asociativos en la cadena de la papa: Villapinzón, provincia de Almeidas, Cundinamarca. Rev. CIFE 13, 204-226.

Sandoval, H. 2013. Se cocina paro papero en 2014. El Espectador, Economía 19 Mayo 2013 - 10:00 pm. En: http://www.elespectador.com/noticias/ economia/articulo-422898-se-cocina-paro-papero-2014; consulta: 2013.

Secretaría de Agricultura y Desarrollo Económico (SADE) - Fedepapa. 2009. Recopilación de la investigación del sistema productivo papa criolla. Convenio SADE 045/06 (Gobernación de Cundinamarca; Federación Colombiana de Productores de Papa, Bogotá.

Villarreal, H.J. 2012. Competitividad: Estrategia para lograrla. Rev. Papa 29, 4-11. 